\title{
THREE CONVEX SETS
}

\author{
MICHEL TALAGRAND ${ }^{1}$
}

\begin{abstract}
We construct a Choquet simplex $K$ such that there is a universally measurable affine function $f$ on $K$, which satisfies the barycentric calculus, and is zero on the set of extreme points, but is not identically zero. We also construct a closed convex bounded set of a Banach space without extreme points, but such that each point is the barycenter of a maximal measure. Finally, we construct a closed bounded set $L$ of $I^{\prime}(\mathbf{R})$ and a maximal measure on $L$ which is supported by a weak Baire set which contains no extreme points.
\end{abstract}

1. Introduction. Let $K$ be a convex compact set and $A(K)$ the space of affine continuous functions on $K$. For a probability measure $\mu$ on $K$, its barycenter $b_{\mu}$ is the unique point in $K$ such that for each $f \in A(K)$, we have

$$
f\left(b_{\mu}\right)=\int f d \mu .
$$

A function $f$ on $K$ is said to satisfy the barycentric calculus if it is $\mu$-measurable for each Radon measure $\mu$ on $K$ and if (1) holds. Each point of $K$ is the barycenter of a maximal measure $\mu$ on $K$ (see $[\mathbf{1}, \mathbf{4}]$ for references about all the claims concerning Choquet's theory) where maximal means maximal for the order

$$
\mu \prec \nu \Leftrightarrow \forall f \text { convex continuous on } K, \quad \mu(f) \leqslant \nu(f) .
$$

A maximal measure is in many respects "very close to the extreme points". For example, it is supported by each $K_{\sigma}$ which contains the extreme points. It is hence a natural question whether a function which satisfies the barycentric calculus is determined by its values on the extreme points (this question is asked in [3]). In the next section we shall construct a counterexample where $K$ is a Choquet simplex.

Now let $E$ be a Banach space and $A$ a closed convex bounded set of $E$. A Radon measure $\mu$ on $A$ is said to be maximal if it is maximal for the order.

$$
\mu \prec \nu \Leftrightarrow \forall f \text { convex continuous on } A, \quad \mu(f) \leqslant \nu(f) .
$$

In $\S 3$, we shall construct a closed convex bounded set $L$ in $l^{1}(\mathbf{R})$, a maximal measure $\mu$ on $L$, and a weak Baire set $B$ with $\mu(B)=1$, but such that $B$ contains no extreme points. So, even though $L$ has the Radon-Nikodym property, it is very different from a weak compact set. This construction settles a problem left open by G. A. Edgar [2]. Also related to the work of Edgar is an example of a closed

Received by the editors April 6, 1982 and, in revised form, February 5, 1983 and April 4, 1983.

1980 Mathematics Subject Classification. Primary 46A55, 46B20.

'This paper was written while the author was visiting the Ohio State University. 
bounded convex set of $c_{0}(\mathbf{R})$ with no extreme points, but such that each point is the barycenter of a maximal Radon measure. The construction of this example is somewhat similar to the construction of the first example.

ACKnowledgement. The author thanks G. A. Edgar and R. Bourgin for stimulating discussions.

2. A big Choquet simplex. Let $L$ be a compact space and $A$ a subspace of $C(L)$ which contains the constants. The construction will follow by repeated use of a constant procedure which we describe now. We denote by $\lambda$ the Lebesgue measure on $[0,1]$. Let $L^{\prime}=L \cup L \times[0,1]$, provided with the "porcupine" topology, i.e. the topology given by the following subbasis of open sets:

$$
\begin{gathered}
\{l\} \times U \text { for } l \in L, U \text { open in }[0,1], \\
V \cup(V \backslash\{l\}) \times[0,1] \text { for } l \in L, V \text { open in } L .
\end{gathered}
$$

Let $j$ be the canonical injection $L \rightarrow L^{\prime}$ and $p: L^{\prime} \rightarrow L$ given by $p(l)=l$ and $p((l, t))=l$.

Let $A^{\prime}$ be the space of $f^{\prime} \in C\left(L^{\prime}\right)$ which satisfy the following conditions:

$$
\begin{gathered}
f^{\prime} \circ j \in A, \\
\forall l \in L, \quad f^{\prime}(l)=\int f^{\prime}(l, t) d \lambda(t),
\end{gathered}
$$

where for simplicity we write $f^{\prime}(l, t)$ instead of $f^{\prime}((l, t))$. Let $i$ be the injection of $A$ in $A^{\prime}$ given by $i(f)=f \circ p$.

Lemma 1. Let $\mu$ be a Radon probability on $L^{\prime}$. If $p(\mu)$ is diffuse, then $\mu(j(L))=1$.

Proof. If $\mu\left(L^{\prime} \backslash L\right)>0$, since $L^{\prime} \backslash L$ is the union of the open sets $\{l\} \times[0,1]$, there exists $l \in L$ with $\mu(\{l\} \times[0,1])>0$. Since $p(\{l\} \times[0,1])=\{l\}$, it shows that $p(\mu)(\{l\})>0$, and proves the lemma.

We also need a condition which will ensure that $A^{\prime}=A\left(K^{\prime}\right)$ for a simplex $K^{\prime}$. Recall that $K$ is a simplex if and only if $A(K)$ satisfies the Riesz interpolation property.

Definition 2. We say that $A$ satisfies the property (*) if, given $f_{1}, f_{2}, g_{1}, g_{2} \in A$, with $f_{1}, f_{2}<g_{1}, g_{2}$, and given $n$ points $x_{1}, \ldots, x_{n} \in L, t_{1}, t_{1}^{\prime}, \ldots, t_{2}, t_{2}^{\prime} \in \mathbf{R}$ with

$$
\forall i \leqslant n, \quad \sup \left(f_{1}\left(x_{i}\right), f_{2}\left(x_{i}\right)\right) \leqslant t_{i}<t_{i}^{\prime} \leqslant \inf \left(g_{1}\left(x_{i}\right), g_{2}\left(x_{i}\right)\right),
$$

there exists $h \in A, f_{1}, f_{2}<h<g_{1}, g_{2}$, and $\forall i \leqslant n, t_{i}<h\left(x_{i}\right)<t_{i}^{\prime}$.

Lemma 3. If $A$ satisfies (*), then $A^{\prime}$ satisfies (*).

Proof. Let $f_{1}^{\prime}, f_{2}^{\prime}, g_{1}^{\prime}, g_{2}^{\prime} \in A^{\prime}$ with $f_{1}^{\prime}, f_{2}^{\prime}<g_{1}^{\prime}, g_{2}^{\prime}$. Let $\varepsilon$ be such that $\inf \left(g_{1}^{\prime}, g_{2}^{\prime}\right)-$ $\sup \left(f_{1}^{\prime}, f_{2}^{\prime}\right)>\varepsilon$. Let $x_{1}, \ldots, x_{n} \in L^{\prime}$. We can assume that $x_{1}, \ldots, x_{m} \in L$ and $x_{m+1}, \ldots, x_{n} \in L^{\prime} \backslash L$ for one $m \leqslant n$. Let $t_{i}, t_{i}^{\prime} \in \mathbf{R}$ such that

$$
\forall i \leqslant n, \quad \sup \left(f_{1}^{\prime}\left(x_{i}\right), f_{2}^{\prime}\left(x_{i}\right)\right) \leqslant t_{i}<t_{i}^{\prime} \leqslant \inf \left(g_{1}^{\prime}\left(x_{i}\right), g_{2}^{\prime}\left(x_{i}\right)\right) \text {. }
$$


It is easily seen from the definition of the topology of $L^{\prime}$ that there is a finite set $F \in L$ such that for $l \notin F$, and for $g$ one of $f_{1}^{\prime}, f_{2}^{\prime}, g_{1}^{\prime}, g_{2}^{\prime}$, one has

$$
\forall t \in[0,1], \quad|g(l)-g(l, t)| \leqslant \varepsilon / 10 .
$$

For $l \in F$, let

$$
t_{l}=\int \sup \left(f_{1}^{\prime}(l, t), f_{2}^{\prime}(l, t)\right) d \lambda(y), \quad t_{l}^{\prime}=\int \inf \left(g_{1}^{\prime}(l, t), g_{2}^{\prime}(l, t)\right) d \lambda(t) .
$$

Since $A$ satisfies (*), there is an $h \in A$ such that if $f_{1}, f_{2}, g_{1}, g_{2}$ denote the restrictions, respectively, of $f_{1}^{\prime}, f_{2}^{\prime}, g_{1}^{\prime}, g_{2}^{\prime}$ to $L$, we have $f_{1}+\varepsilon / 4, f_{2}+\varepsilon / 4<h<g_{1}-$ $\varepsilon / 4, g_{2}-\varepsilon / 4$ and $\forall i \leqslant n, t_{i}<h\left(x_{i}\right)<t_{i}^{\prime} ; \forall l \in L, t_{1}<h(l)<t_{l}^{\prime}$. Let $F^{\prime}$ be the finite set of those $l \in L$ such that $\{l\} \times[0,1]$ contains one $x_{i}(m<i \leqslant n)$. For $l \notin F \cup F^{\prime}$ we set $h^{\prime}(l, t)=h(l)$. For $l \in F \cup F^{\prime}$ it is easily seen that there is a $u \in C([0,1])$ such that

$$
\begin{gathered}
\forall t \in[0,1], \quad f_{1}^{\prime}(l, t), f_{2}^{\prime}(l, t)<u(t)<g_{1}^{\prime}(l, t), g_{2}^{\prime}(l, t), \\
\int u(t) d \lambda(t)=h(l), \\
\forall m<i \leqslant n \text { such that } x_{i}=\left(l, a_{i}\right), \quad t_{i}<u\left(a_{i}\right)<t_{i}^{\prime},
\end{gathered}
$$

and we set $h^{\prime}(l, t)=u(t)$. It is straightforward to check that $h^{\prime} \in A^{\prime}$ and satisfies all the requirements. The lemma is proved.

We now proceed to the construction. Let $\Omega$ be the first uncountable ordinal. For $\alpha \leqslant \Omega$, we shall construct compact spaces $L_{\alpha}$ and subspaces $A_{\alpha}$ of $C\left(L_{\alpha}\right)$ which satisfy (*). For $\beta<\alpha$, there is a map $p_{\beta, \alpha}$ from $L_{\alpha}$ into $L_{\beta}$, and an injection $j_{\alpha, \beta}$ of $L_{\beta}$ into $L_{\alpha}$, with $p_{\beta, \alpha} \circ j_{\alpha, \beta}$ being the identity of $L_{\beta}$.

The construction goes as follows. We start with $L_{0}=\{0\}, A_{0}=C\left(L_{0}\right)=\mathbf{R}$. Suppose the construction has been done for each $\alpha<\gamma$.

lst case. $\gamma$ is of the form $\alpha+1$. Then we set $L_{\gamma}=L_{\alpha}^{\prime}, A_{\gamma}=A_{\alpha}^{\prime}$. If $j: L \rightarrow L^{\prime}$ and $p: L^{\prime} \rightarrow L$ are the natural injection and projection, for $\beta<\alpha$ we set $j_{\gamma, \beta}=j \circ j_{\alpha, \beta}$ and $p_{\beta, \gamma}=p_{\gamma, \alpha} \circ p$. From Lemma $3, A_{\gamma}$ satisfies $(*)$ and the construction is completed in this case.

2nd case. $\gamma$ is a limit ordinal. Then $L_{\gamma}$ is the projective limit of the $\left(L_{\alpha}\right)_{\alpha<\gamma}$. Mappings $j_{\gamma, \beta}$ and $p_{\beta, \gamma}$ are defined in the obvious way. For $f \in A_{\beta}$, let $i_{\beta}(f)=f \circ p_{\beta, \gamma}$ and let $A_{\gamma}=\cup_{\beta<\gamma} i_{\gamma}\left(A_{\beta}\right)$. All the verifications are straightforward, so the construction is completed.

Let $L=L_{\Omega}, A=\overline{A_{\Omega}}$. Since $A_{\Omega}$ satisfies (*), $A$ also satisfies (*). Let $K=\left\{h \in A^{*}\right.$, $h \geqslant 0, h(u)=1\}$, where $u(l)=1 \forall l \in L$. Then, as is well known, $A=A(K)$. Moreover, $L$ identifies as a subset of $K$, which contains the extreme points. Since $A$ satisfies (*), $A$ satisfies the Riesz interpolation property, so $K$ is a Choquet simplex. For simplicity, we identify each $L_{\alpha}$ to a subset of $L$, and we denote by $p_{\alpha}$ the natural projection of $L$ onto $L_{\alpha}$.

Let $K_{\alpha}$ be the closed convex hull of $L_{\alpha}$. 
Lemma 4. (a) The set of extreme points of $K$ is $E=L \backslash \cup_{\beta<\Omega} L_{\beta}$.

(b) For a diffuse Radon probability $\mu$ on $L$, there exists $\alpha$ with $\mu\left(L_{\alpha}\right)=1$.

(c) Each Radon probability $\mu$ on $L$ can be written in a unique way, $\mu=\mu^{\prime}+\mu^{\prime \prime}$ where there is an $\alpha$ for which $\mu^{\prime}\left(L_{\alpha}\right)=\mu^{\prime}(L)$, and $\mu^{\prime \prime}$ is atomic, $\mu^{\prime \prime}(E)=\mu^{\prime \prime}(L)$. In particular, $E$ is universally measurable.

(d) Let $\mu_{1}$ and $\mu_{2}$ be probabilities on L. If $\mu_{1}(f)=\mu_{2}(f)$ for each $f \in A(K)$, then $\mu_{1}$ and $\mu_{2}$ have the same restriction to $E$.

Proof. (a) If $a \in L_{\beta}$, then condition (4) shows that $a$ is the barycenter of a copy of Lebesgue measure on $\{a\} \times[0,1]$, so $a$ is not extremal. It follows that each extreme point belongs to $E$. The converse follows from (d).

(b) Since $\mu$ is diffuse, for each $n$ there is an $\alpha_{n}<\Omega$ such that $p_{\alpha_{n}}(\mu)$ has no atoms of weight $\geqslant n^{-1}$. If $\alpha=\sup \alpha_{n}$, then $p_{\alpha}(\mu)$ is diffuse. From Lemma 2 and by induction one proves that for $\gamma>\alpha$ we have $p_{\gamma}(\mu)\left(j_{\mu, \alpha}\left(L_{\alpha}\right)\right)=1$, so $\mu\left(L_{\alpha}\right)=1$.

(c) Let $\mu$ be a Radon probability on $L$, and $\alpha<\Omega$ such that $\mu\left(K_{\alpha}\right)=$ $\sup _{\beta<\Omega} \mu\left(K_{\beta}\right)$. Let $\mu^{\prime}$ be the restriction of $\mu$ to $L_{\alpha}$, and $\mu^{\prime \prime}=\mu-\mu^{\prime}$. We have $\mu^{\prime}\left(L_{\beta}\right)=0$ for each $\beta$. It follows from (b) that $\mu^{\prime}$ is atomic and then, of course, is supported by $E$.

(d) It is enough to show that if $a \in E$ then $\mu_{2}(\{a\}) \geqslant \mu_{1}(\{a\})$. Let

$$
H=\left\{\alpha<\Omega ; p_{\alpha+1}(a) \in L_{\alpha+1} \backslash L_{\alpha}\right\} .
$$

If for $\alpha \geqslant \beta$ we have $\alpha \notin H$, we show by induction that $p_{\alpha}(a) \in j_{\alpha, \beta}\left(L_{\beta}\right)$, so $a \in L_{\beta}$, a contradiction. It follows that $H$ is unbounded.

Let $\alpha \in H$. Let $p_{\alpha+1}(a)=\left(l^{\prime}, t^{\prime}\right)$, where $l^{\prime} \in L_{\alpha}$. Consider a sequence $\left(u_{n}\right)$ of $C([0,1])$ with $\int u_{n}(t) d t=0$ and $u_{n}(t) \rightarrow 1$ if $t=t^{\prime}$, and $u_{n}(t) \rightarrow 0$ otherwise. Let $f_{n} \in A_{\alpha+1}$ be given by $f_{n}(l)=0$ for $l \in L_{\alpha}, f_{n}(l, t)=0$ if $l \neq l^{\prime}, f_{n}\left(l^{\prime}, t\right)=u_{n}(t)$. Since

$$
p_{\alpha+1}\left(\mu_{1}\right)\left(f_{n}\right)=p_{\alpha+1}\left(\mu_{2}\right)\left(f_{n}\right)
$$

for each $n$, this forces $p_{\alpha+1}\left(\mu_{2}\right)(l, t) \geqslant p_{\alpha+1}\left(\mu_{1}\right)(l, t)$, i.e. $\mu_{2}\left(p_{\alpha+1}^{-1}\left(p_{\alpha+1}(a)\right)\right) \geqslant$ $\mu_{1}(\{a\})$. But letting $\alpha$ grow in $H$ to $\Omega$, one gets $\mu_{2}(\{a\}) \geqslant \mu_{1}(\{a\})$. The lemma is proved.

For $a \in K$, property (d) shows that we can define $f(a)=\mu(L \backslash E)$, where $\mu$ is any measure on $L$ of barycenter $a$.

If $a \in E$, we have $f(a)=0$, since $\delta_{a}$ represents $a$. If $a \in K_{\alpha}, a$ is the barycenter of a measure on $L_{\alpha}$, so $f(a)=1$.

It remains to show that $f$ satisfies the barycentric calculus. Let $\nu$ be a Radon probability on $K$. The map $\varphi$ from $M_{+}^{1}(L)$ onto $K$, which sends a measure on its barycenter, is continuous; so there is a Radon probability $\theta$ on $M_{+}^{1}(L)$ such that $\nu=\varphi(\theta)$. Let $\bar{\theta}$ be the probability on $L$ given by

$$
\bar{\theta}(g)=\int_{M_{+}^{1}(L)} \mu(g) d \theta(\mu)
$$


for $g \in C(L)$. If $g \in A(K)$, we have

$$
\begin{aligned}
g\left(b_{\theta}^{-}\right) & =\bar{\theta}(g)=\int \mu(g) d \theta(\mu)=\int g\left(b_{\mu}\right) d \theta(\mu) \\
& =\int g(x) d \nu(x)=g\left(b_{\nu}\right)
\end{aligned}
$$

so $b_{\theta}^{-}=b_{\nu}$.

There exist $\alpha$ and a countable set $D \subset E$ such that $\bar{\theta}\left(K_{\alpha} \cup D\right)=1$. Since $(* *)$ holds also for upper semicontinuous functions, we have $\mu\left(K_{\alpha} \cup D\right)=1 \theta$-almost everywhere, whence it follows that $\mu\left(K_{\alpha}\right)=\mu(L \backslash E) \theta$-a.e. so we have

$$
\begin{aligned}
f\left(b_{\nu}\right) & =f\left(b_{\theta}^{-}\right)=\theta(L \backslash E)=\theta\left(K_{\alpha}\right)=\int \mu\left(K_{\alpha}\right) d \theta(\mu) \\
& =\int \mu(L \backslash E) d \theta(\mu)=\int f\left(b_{\mu}\right) d \theta(\mu)=\int f \circ \varphi(\mu) d \theta(\mu) .
\end{aligned}
$$

The proof shows that $f \circ \varphi$ is $\theta$-measurable. It follows that $f$ is $\nu$-measurable and $\int f \circ \varphi(\mu) d \theta(\mu)=\int f(x) d \nu(x)$, which finishes the proof.

THEOREM 5. There exists a Choquet simplex $K$ and an affine $f$ on $K$ which satisfies the barycentric calculus, and is not identically zero, but vanishes on the extreme points.

3. A big convex set. The construction here will also use the same standard process at each step.

We denote by $K$ the unit disk of $\mathbf{R}^{2}$, and by $\lambda$ the uniform measure on the unit circle. Then $\lambda$ is maximal in $K$, and its barycenter is zero. A point $k \in K$ will be denoted by $(k(1), k(2))$.

Let $A$ be a bounded closed convex subspace of $c_{0}(\Gamma)$ with $0 \in A$. Let $\Gamma^{\prime}=\Gamma \cup$ $(\{1,2\} \times A)$. We consider $c_{0}(\Gamma)$ as a subspace of $c_{0}\left(\Gamma^{\prime}\right)$. For $a \in A, k \in K$, we define $a * k$ by

$$
\begin{aligned}
a * k\left(\gamma^{\prime}\right) & =a(\gamma) & & \text { if } \gamma^{\prime}=\gamma \in \Gamma \\
a * k\left(\gamma^{\prime}\right) & =k(n) & & \text { if } \gamma^{\prime} \text { is of the form }(n, a), n \in\{1,2\} \\
& =0 & & \text { otherwise. }
\end{aligned}
$$

Notice that $a=a * 0$.

We denote by $A^{\prime}$ the closed convex hull of the points $a * k$ for $a \in A, k \in K$. We denote by $p$ the canonical projection of $c_{0}\left(\Gamma^{\prime}\right)$ onto $c_{0}(\Gamma)$, and by $p_{a}$ the map from $c_{0}\left(\Gamma^{\prime}\right)$ to $\mathbf{R}^{2}$ given by $p_{a}(x)=(x((1, a)), x((2, a)))$. Notice that $A=p\left(A^{\prime}\right)$.

Lemma 6. $A^{\prime}=\left\{\sum_{a \in A} \alpha_{a} a * k_{a} ; \alpha_{a} \geqslant 0, \Sigma \alpha_{a} \leqslant 1, k_{a} \in K\right\}$.

Proof. Let $p$ be the positive part of the unit ball of $l^{\prime}(A)$. The map $\varphi$ : $P \times K^{A} \rightarrow c_{0}\left(\Gamma^{\prime}\right)$ which sends $\left(\alpha_{a}\right)_{a \in A} \times\left(k_{a}\right)_{a \in A}$ to $\sum_{a \in A} \alpha_{a} a * k_{a}$ is continuous when $l^{1}(A)$ is provided with the $w^{*}$-topology, $K^{A}$ with the product topology, $l^{1}(A) \times K^{A}$ with the product topology and $c_{0}\left(\Gamma^{\prime}\right)$ with the weak topology. Hence its image is weak compact. Since it is convex it is norm closed. The claim follows. 
Lemma 7. Let $\mu$ be a maximal diffuse measure on $A$. Then $\mu$, seen as a measure on $A^{\prime}$, is maximal.

Proof. Let $\nu$ be a measure on $A^{\prime}$ with $\nu \prec \nu$. Since $\mu=p(\mu) \prec p(\nu)$, we have $p(\nu)=\mu$.

Suppose, for contradiction, that $\nu\left(A^{\prime} \backslash A\right)>0$. Since $\nu$ is a Radon measure, the intersection of a family of closed sets of full measure is of full measure. Since $A=A^{\prime} \cap c_{0}(\Gamma)$, there exists $a$ and $\varepsilon$ such that $\nu(U)>0$, where $U=\left\{x \in A^{\prime}\right.$; $\left.\left\|p_{a}(x)\right\|>\varepsilon\right\}$. Let $\nu^{\prime}$ be the normalisation of the restriction of $\nu$ to $U$. For $x \in U$, $x=\sum \alpha_{a} a * k_{a}$, we have $p_{a}(x)=\alpha_{a} k_{a}$, so $\left\|p_{a}(x)\right\|>\varepsilon$, so $\alpha_{a}>\varepsilon$, so $\sum_{h \neq a} \alpha_{h}<1-\varepsilon$. It follows that $p(x) \in B=\varepsilon a+(1-\varepsilon) A$; so $p\left(\nu^{\prime}\right)(B)=1$.

Since $\nu^{\prime} \ll \nu,\left[2\right.$, Theorem 5-1] shows that $\nu^{\prime}$ is maximal. However, $B \backslash\{a\}$ is a movable set in the sense of [2], since for $b \in B$ we have

$$
b=1 / 2((\gamma b+(1-\gamma) a)+(2-\gamma) b-(1-\gamma) a),
$$

where $\gamma=(1-2 \varepsilon) /(1-\varepsilon)$; this contradiction shows that $\nu(A)=1$, so $p(\nu)=\nu=$ $\mu$.

LEMMA 8. Each point of $a$ is the barycenter of a maximal diffuse measure $\mu$ on $A$.

Proof. Let $\lambda_{a}$ be the image measure of $\lambda$ under the map $k \rightarrow a * k$. It is a diffuse measure, with barycenter $a$. Moreover, it is straightforward from.Lemma 6 to check that each point, $a * k$ with $\|k\|=1$, is extremal; so $\lambda_{a}$ is maximal.

We now have the tools for a first example:

THEOREM 9. There exist a closed bounded convex set $L$ of $l^{\prime}(\mathbf{R})$, a maximal measure $\mu$ on $L$, and a weak Baire set $B$ of $l^{\prime}(\mathbf{R})$ which supports $\mu$ but contains no extreme points.

Proof. We make the above construction with $A=K, \Gamma=\{1,2\}$ and set $L=A^{\prime}$. Then Lemma 6 shows that $L$ is a subset of $l^{1}\left(\Gamma^{\prime}\right)$, and since the $l^{1}$-norm is finer than the $c_{0}$-norm, it is closed in $l^{\prime}$.

From Lemma 7, the measure $\lambda$ on $A$ is maximal on $A^{\prime}$. Let $I=\left\{(n, a) \in \Gamma^{\prime}\right.$, $a \in A, n=1,2\}$ and $B=\left\{x \in l^{\prime}\left(\Gamma^{\prime}\right) ; x(i)=0, \forall i \in I\right\}$. Then $A \subset B$, so $\lambda(B)=1$. Moreover, $B$ contains no extreme point since each point of $a$ is the barycenter of the measure $\lambda_{a}$.

Finally, since $l^{1}(I)$ is a subspace of $l^{\infty}$, its dual is weak*-separable, so there is a sequence $y_{n} \in l^{\prime}\left(\Gamma^{\prime}\right)^{*}$ such that $B=\left\{x ; \forall n, y_{n}(x)=0\right\}$, so $B$ is a weak Baire set. The proof is complete.

We can now proceed to the construction of the last example. For $\alpha<\Omega$ we construct sets $\Gamma_{\alpha}$, with card $\Gamma_{\alpha}=$ card $\mathbf{R}$, and closed convex sets $A_{\alpha} \subset c_{0}\left(\Gamma_{\alpha}\right)$ such that for $\beta<\alpha$, we have $\Gamma_{\beta} \subset \Gamma_{\alpha}$, and $A_{\beta} \subset A_{\alpha}$ (where $c_{0}\left(\Gamma_{\beta}\right)$ is identified to a subspace of $\left.c_{0}\left(\Gamma_{\alpha}\right)\right)$ in the following way. We set $A_{0}=K, \Gamma_{0}=\mathbf{N}$. If the construction has been done for $\alpha$, we set $\Gamma_{\alpha+1}=\Gamma_{\alpha}^{\prime}$ and $A_{\alpha+1}=A_{\alpha}^{\prime}$. If the construction has been done for each $\alpha<\gamma$, and $\gamma$ is limit, then $\Gamma_{\gamma}=\cup_{\alpha<\gamma} \Gamma_{\alpha}$ and $A_{\gamma}$ is the closure of $\bigcup_{\alpha<\gamma} A_{\alpha}$. 
We set $A=\cup_{\alpha<\Omega} A_{\alpha}$. Since each sequence in $A$ is contained in one of the $A_{\alpha}, A$ is closed. Let $a \in A$. Then there is an $\alpha$ such that $a \in A_{\alpha}$. From Lemma 7, $a$ is the barycenter of a maximal probability $\mu$ on $A_{\alpha+1}^{\prime}$. Let $\nu$ be a probability on $\mu$ with $\mu \prec \nu$. Using Lemma 6, one shows by induction over $\gamma$ that if $p_{\gamma}$ denotes the natural projection of $c_{0}(\Gamma)$ onto $c_{0}\left(\Gamma_{\gamma}\right)$, then $p_{\gamma}(\nu)=\mu$. It follows that $\nu=\mu$, so $\mu$ is maximal on $A$. This also shows that $A$ has no extreme points.

THEOREM 10. There is a convex bounded closed set of $c_{0}(\mathbf{R})$ which has no extreme points, but such that each point is the barycenter of a maximal measure.

\section{REFERENCES}

1. (j. Choquet, Lectures on analysis, Benjamin, New York, 1969.

2. G. A. Edgar, Extremal integral representation, J. Funct. Anal. 23 (1976), 145-165.

3. R. Haydon, Some more characterizations of Banach spaces containing $l_{1}$, Math. Proc. Cambridge Philos. Soc. 80 (1976), 269-276.

4. R. R. Phelps, Lecture on Choquet's theorem, Van Nostrand, Princeton, N. J., 1966.

Equipe d'Analyse-Tour 46, Universite Paris Vi, 4 Place Jussieu, 75230 Paris Cedex 05, France 